\title{
The potential diagnostic use of clinical characteristics and mitochondrial DNA copy numbers of peripheral blood and ovarian tissue in polycystic ovary syndrome (PCOS) patient
}

\section{Yi Song}

The Chinese University of Hong Kong

\section{Yan Deng}

The Chinese University of Hong Kong Jacqueline Chung

The Chinese University of Hong Kong

Tin Chiu Li

The Chinese University of Hong Kong

\section{Chi Chiu Wang}

The Chinese University of Hong Kong

Hu Li

Pan Yu Central Hospital

\section{Tao Yang}

Fudan University Pudong Medical Center

Yi Sui

The First Affiliated Hospital of Sun Yat-sen University

\section{Tao Tang ( $\boldsymbol{\nabla}$ tangtao@cuhk.edu.hk)}

The Chinese University of Hong Kong

\section{Research Article}

Keywords: PCOS, mtDNA, copy number, diagnosis

Posted Date: October 31st, 2022

DOI: https://doi.org/10.21203/rs.3.rs-1046361/v2

License: (c) (1) This work is licensed under a Creative Commons Attribution 4.0 International License. Read Full License 


\section{Abstract}

Polycystic ovary syndrome (PCOS) is a complex, heterogeneous syndrome of uncertain etiology characterized by hyperandrogenemia, hyperinsulinemia, chronic anovulation, and polycystic ovaries. Recent studies showed that the abnormalities of mitochondrial metabolism are related to PCOS. We hypothesized that mtDNA copy number is an important marker that can reflect mitochondrial function. In this study, 135 PCOS patients and 57 age-matched healthy participants were studied. Mitochondrial DNA copy number in peripheral blood and PCOS ovarian tissues, and some clinical parameters were assessed. From the single factor analysis, we can find some clinical parameters are different between PCOS and healthy women and the mitochondrial DNA copy numbers in peripheral blood in PCOS women were significantly lower than in healthy women. We also found that there was no correlation between mtDNA copy numbers in peripheral blood and in ovarian tissue. After multiple logistic regressions, we identified the occurrence of PCOS was significantly positively correlated with BMI and pulse, and negatively correlated with mitochondrial DNA copy numbers in peripheral blood. We also found the decreased mtDNA copy numbers in PCOS patients are independent of these clinical parameters. We constructed the ROC curve based on these risk factors and found if they have potential to predict the patient's outcome. In conclusion, the changes in mtDNA copy number and some clinical parameters may provide new ideas for PCOS diagnosis. More studies are necessary for further validation of their use in PCOS diagnosis.

\section{Introduction}

Polycystic ovary syndrome (PCOS) is one of the most common reproductive and endocrine abnormalities, which accounts for about $75 \%$ of anovulatory infertility and affects up to $17.8 \%$ of women in the reproductive age (13-45 years old) [1-3]. It is a complex, heterogeneous syndrome of uncertain etiology characterized by hyperandrogenemia, hyperthecosis, hyperinsulinemia, chronic anovulation, and polycystic ovaries [4-10]. PCOS is also associated with metabolic disturbances including dyslipidemia, insulin resistance, and type 2 diabetes mellitus (DM2) [10-13]. Recently, elevated oxidative stress has been reported in PCOS.

Mitochondria play an important role in cellular energy supply and regulate the production of reactive oxygen species (ROS) $[14,15]$. The ROS can cause significant oxidative stress in the mitochondria, resulting in the increased mutation rates of mitochondrial DNA (mtDNA), which in turn lead to enzymatic abnormalities and further oxidative stress [16]. Recently, a study demonstrated that the expression levels of genes related to mitochondrial oxidative phosphorylation were reduced in skeletal muscle in insulinresistant PCOS patients [17]. Damage or dysfunction in mitochondria may lead to cellular metabolic activity reduction [18] and mtDNA copy numbers can be used for the measurement of the mass of mitochondria. Lee et al. observed that decreased mtDNA copy number was found in peripheral blood of Korean PCOS patients when compared to controls and it was independent of insulin resistance or other metabolic factors [19]. Another study showed treatment with metformin for one year caused a timedependent decrease in mtDNA copy number in patients with PCOS [20]. Also, this study showed that the change in mtDNA copy number was positively correlated to the serum level of total testosterone, however 
other studies did not show any correlations with most of the clinical indexes. From the generalized estimating equations (GEE) models, only testosterone was significantly associated with mtDNA after metformin treatment [20].

Based on the above studies, we propose that the mtDNA copy number is a marker of mitochondrial metabolism, and abnormalities in the mtDNA copy number are related to PCOS. In this study, we further investigated mtDNA copy numbers in PCOS of Chinese people with larger population samples. mtDNA copy numbers not only in peripheral blood but also in PCOS ovarian tissues were detected by real-time polymerase chain reaction. The correlation between mtDNA copy number and metabolic parameters was assessed. We also include some clinical parameters in our studies to find out if they are correlated with mtDNA or PCOS.

\section{Results}

\subsection{Clinical characteristics of PCOS patients and control participants}

Clinical characteristics of the PCOS patients and control participants were summarized in Table 1. The median age was 33 years for both groups. PCOS group showed an average BMI of $26.9 \mathrm{~kg} / \mathrm{m}^{2}$, which was greater than that of the control group $\left(B M I=23.1 \mathrm{~kg} / \mathrm{m}^{2}\right)$ and was considered overweight. Additionally, systolic BP, pulse and ALT in the PCOS group were significantly higher and HDL-Cholesterol in the PCOS group was lower than that of the control group. All the patients had normal fasting glucose levels. 
Table 1

Clinical characteristics of PCOS patients and healthy controls

\begin{tabular}{|c|c|c|c|}
\hline Index & $\operatorname{PCOS}(n=135)$ & Control $(n=57)$ & $P$ value \\
\hline Age $(y)$ & $33.1 \pm 5.3$ & $34.2 \pm 9.3$ & .438 \\
\hline $\mathrm{BMI}\left(\mathrm{kg} / \mathrm{m}^{2}\right)$ & $26.9 \pm 5.3$ & $23.1 \pm 3.4$ & .000 \\
\hline Systolic BP (mmHg) & $126.2 \pm 18.1$ & $120.2 \pm 17.5$ & .034 \\
\hline Diastolic BP (mmHg) & $76.9 \pm 14.0$ & $74.2 \pm 12.5$ & .205 \\
\hline Pulse (times/min) & $86.8 \pm 15.7$ & $78.4 \pm 12.1$ & .000 \\
\hline Fasting glucose (mmol/L) & $5.1 \pm 0.8$ & $5.0 \pm 0.7$ & .287 \\
\hline Urea (mmol/L) & $4.0 \pm 1.0$ & $4.0 \pm 1.5$ & .573 \\
\hline Creatinine $(\mu \mathrm{mol} / \mathrm{L})$ & $60.0 \pm 9.7$ & $60.1 \pm 16.2$ & .937 \\
\hline ALP (U/L) & $60.0 \pm 20.6$ & $68.6 \pm 40.4$ & .113 \\
\hline $\operatorname{ALT}(\mathrm{U} / \mathrm{L})$ & $26.4 \pm 21.1$ & $20.0 \pm 9.9$ & .004 \\
\hline Cholesterol (mmol/L) & $4.9 \pm 0.9$ & $5.3 \pm 1.1$ & .085 \\
\hline HDL-Cholesterol (mmol/L) & $1.4 \pm 0.4$ & $1.7 \pm 0.3$ & .001 \\
\hline Triglycerides (mmol/L) & $1.2 \pm 0.7$ & $1.0 \pm 0.7$ & .097 \\
\hline LDL-Cholesterol (mmol/L) & $3.0 \pm 0.8$ & $3.1 \pm 1.1$ & .456 \\
\hline
\end{tabular}

\subsection{Mitochondrial DNA copy number in peripheral blood and ovary tissues}

As the original values of the relative mtDNA copy number were not normally distributed, logarithmic transformation was used when analyzing the mtDNA copy number of PCOS patients and control subjects. Compared with healthy control, the mtDNA copy number was markedly lower in PCOS patients (Fig. 1, $P<0.05$ ).

Furthermore, the mtDNA copy number of 26 ovarian tissues and 203 blood samples was analyzed and coefficients (R) and P values were calculated using the Pearson correlation model. As shown in Table 2, there was no correlation between peripheral blood mtDNA copy number and ovarian tissue mtDNA copy number $(R=-0.134 ; P=0.512)$. 
Table 2

Correlation of peripheral blood mtDNA copy numbers and ovarian tissue mtDNA copy numbers

\begin{tabular}{|llll|}
\hline & & PCOS blood & PCOS tissue \\
\hline PCOS blood & Pearson Correlation & 1 & -.134 \\
\cline { 2 - 4 } & Sig.(2-tailed) & & .512 \\
\cline { 2 - 4 } & Sample size(N) & 203 & 26 \\
\hline PCOS tissue & Pearson Correlation & -.134 & 1 \\
\cline { 2 - 4 } & Sig.(2-tailed) & .512 & \\
\cline { 2 - 4 } & Sample size(N) & 26 & 26 \\
\cline { 2 - 4 } & &
\end{tabular}

We also analyzed the mtDNA copy number in the 26 patients' ovarian tissues and their corresponding blood samples. As shown in Table 3, there was also no correlation between peripheral blood mtDNA copy number and ovarian tissue mtDNA copy number $(R=0.142 ; P=0.490)$.

Table 3

Correlation of mtDNA copy numbers in ovarian tissue and its corresponding peripheral blood

\begin{tabular}{|llll|}
\hline \multirow{2}{*}{ PCOS blood } & & PCOS blood & PCOS tissue \\
\cline { 2 - 4 } & Pearson Correlation & 1 & .142 \\
\cline { 2 - 4 } & Sig.(2-tailed) & & .490 \\
\cline { 2 - 4 } PCOS tissue & Sample size(N) & 26 & 26 \\
\cline { 2 - 4 } & Pearson Correlation & .142 & 1 \\
\cline { 2 - 4 } & Sig.(2-tailed) & .490 & \\
\cline { 2 - 4 } & Sample size(N) & 26 & 26 \\
\cline { 2 - 4 } \\
\cline { 2 - 4 }
\end{tabular}

In order to eliminate the confounding factors, we used multiple logistic regressions to determine the significant risk factors for PCOS patients. The odds ratios $(95 \% \mathrm{Cl})$ for PCOS by $\mathrm{BMI}$, pulse and logtransformed mtDNA copy number were 1.634 (1.261-2.117), 0.983 (0.923-1.046) and 0.001 (0.0000.033) respectively (Table 4), which indicated PCOS was positively correlated with BMI and pulse, while negatively correlated with mtDNA copy number. 
Table 4

Significant risk factors for PCOS patients determined by using a multiple logistic regression model

\begin{tabular}{|lccc|}
\hline Variable & Odd ratio & $95 \% \mathrm{Cl}$ & P value \\
\hline BMI $\left(\mathrm{kg} / \mathrm{m}^{2}\right)$ & 1.634 & $1.261-2.117$ & .000 \\
\hline Pulse (times/min) & 0.983 & $0.923-1.046$ & .000 \\
\hline log-transformed mtD NA copy number in peripheral blood & 0.001 & $0.000-0.033$ & .000 \\
\hline Data shown as OR and 95\% Cl. P value are calculated by multiple logistic regression. & \\
\hline
\end{tabular}

Furthermore, we wanted to find if there is any correlation between clinical characteristics and mtDNA copy numbers. In PCOS patients, the mtDNA copy number was not correlated with all the above clinical characteristics. In the control group, there was no correlation between mtDNA copy number and these clinical characteristics (Table 5).

Table 5

Correlation of log-transformed peripheral blood mtDNA copy number and biochemical indexes in PCOS patients and control group

\begin{tabular}{|c|c|c|c|c|}
\hline & \multicolumn{4}{|c|}{ Log-transformed mtDNA copy number } \\
\hline & \multicolumn{2}{|c|}{$\operatorname{PCOS}(n=135)$} & \multicolumn{2}{|c|}{ Control $(n=57)$} \\
\hline & $\mathrm{R}$ & $P$ value & $\mathrm{R}$ & $P$ value \\
\hline Systolic BP (mmHg) & 0.085 & .328 & -0.265 & .069 \\
\hline Diastolic BP (mmHg) & 0.152 & .078 & -0.221 & .130 \\
\hline Pulse (times/min) & 0.134 & .121 & 0.253 & .131 \\
\hline Fasting glucose (mmol/L) & -0.013 & .880 & -0.041 & .797 \\
\hline Urea (mmol/L) & -0.070 & .421 & -0.184 & .217 \\
\hline Creatinine $(\mu \mathrm{mol} / \mathrm{L})$ & -0.0105 & .227 & -0.105 & .482 \\
\hline ALP (U/L) & 0.140 & .106 & -0.116 & .431 \\
\hline $\mathrm{ALT}(\mathrm{U} / \mathrm{L})$ & -0.023 & .795 & -0.162 & .299 \\
\hline Cholesterol (mmol/L) & 0.034 & .699 & -.355 & .064 \\
\hline HDL-Cholesterol (mmol/L) & -0.138 & .111 & 0.353 & .065 \\
\hline Triglycerides (mmol/L) & 0.087 & .317 & 0.022 & .935 \\
\hline LDL-Cholesterol (mmol/L) & 0.104 & .230 & -0.007 & .978 \\
\hline
\end{tabular}


To summarize all the results above, we constructed the ROC curve to further investigate if these risk factors can predict the patient's outcome. This ROC curve included three factors: BMI $\left(\mathrm{kg} / \mathrm{m}^{2}\right)$, pulse (times/min) and log-transformed mtDNA copy number in peripheral blood. The Area under the ROC curve is 0.933 and $P=0.000$ (Fig. 2). It means these three risk factors make sense for the PCOS diagnosis.

\section{Discussion}

Polycystic ovary syndrome is an endocrine disease that affects female reproduction. It often exhibits hyperandrogenemia, insulin resistance, and low inflammation. It also increases the risk of type 2 diabetes, metabolic syndrome, hypertension and blood lipids. So far, the molecular mechanism of PCOS is still unclear [21]. More and more studies have focused on oxidative stress caused by mitochondrial dysfunction, which has a negative impact on the development of follicles, indicating that mitochondrial dysfunction plays an important role in the development of PCOS [22, 23]. In recent years, abnormal mtDNA copy numbers and mitochondrial gene mutations in PCOS patients came to the focus of research. In an Iraqi study, a variety of mutations were observed in the mitochondrial transfer RNA in the PCOS group and the mtDNA copy number of this group was lower, whether have diabetes or not [24]. Similar studied were also conducted and discovered the variants of the mitochondrial genome and lower mtDNA copy number of PCOS subjects [25]. In a Han Race family study, the members have inherited insulin resistance and the third generation exhibited PCOS. Analysis of the mtDNA copy number and sequencing data showed that the patients had a mutation in the transfer RNA gene and a lower copy number [26].

Consistently, in this study, we observed the same trend. MtDNA copy number of the PCOS group was significantly lower than that of the control group in peripheral blood. The multiple logistic regression results showed that mtDNA copy number was negatively correlated with PCOS, and BMI and pulse were positively correlated with PCOS. Based on these results, we tried to construct the ROC curve to find if these factors have the diagnostic potential for PCOS. The ROC curve showed that these factors can suggest the PCOS occurrence to some degree. On the other hand, PCOS is associated with cardiovascular diseases $[27,28]$. Analysis of clinical characteristics showed that the systolic BP and pulse were greater in PCOS patients compared with the healthy subjects. As the PCOS patients and control subjects are agematched, the effect of age can be ignored. Since the BMI was not matched between these two groups and it is a crucial factor for cardiovascular diseases, in this study, we cannot prove with strong evidence that PCOS is correlated with cardiovascular diseases.

At present, the molecular mechanism of the pathogenesis of PCOS is still unknown and oxidative stress has been considered as one of the inducing factors. Mitochondria are the main place where ROS are produced and the hub of metabolic activities [29], so the role of mitochondria in the pathogenesis of PCOS cannot be ruled out. Unlike nuclear DNA, mtDNA does not have protective histones and is continuously exposed to the endogenous ROS generated nearby, thus it is more susceptible to environmental carcinogens. The mechanism for lower peripheral mtDNA copy number in PCOS patients is unknown and we are not sure it is the reason or the result of PCOS. Different from the other studies that 
only detecting peripheral samples, mtDNA copy numbers in PCOS ovarian tissue were also detected in this study. Most strikingly, there was no correlation between peripheral mtDNA copy number and tissue mtDNA copy number. Changes in peripheral blood makers do not reflect changes in tissues. This gave us a hint that peripheral mtDNA copy number changes in PCOS may be related to the immune system as the peripheral mtDNA comes from leukocytes. Some studies also found some risk factors which were related to mtDNA copy number in other diseases, including age [31], smoking, and PM 2.5 exposure levels [32]. It was also reported a low level of progesterone causes overstimulation of the immune system, thus producing more estrogen which leads to the accumulation of autoantibodies in PCOS [30]. This opens a new chapter for mtDNA research in PCOS.

This study had some limitations. First, the sample size for the control group is not as large as the PCOS group, as it was hard to recruit healthy women who were willing to join our study. Second, there was no insulin data for all the participants. Third, normal ovarian tissues were not obtained in this study. We could not compare tissue mtDNA copy numbers between the PCOS and the control group. In the future study, larger population samples as well as, "normal ovarian tissues", are necessary.

In conclusion, we reported decreased peripheral mtDNA copy number in PCOS patients is independent of biochemical markers. Future studies in these circumstances may uncover the mechanism of PCOS, thereby helping to identify early biomarkers and develop strategies to reduce the risk of the onset of PCOS in women.

\section{Materials And Methods}

\subsection{Patient recruitment and sample collection}

This study was approved by the Research Ethics Committee of the Joint Chinese University of Hong Kong - New Territories East Cluster Clinical Research Ethics Committee and all protocols were performed in accordance with the relevant guidelines and regulations. A total of 203 women who had been diagnosed with PCOS based on the Rotterdam (2003) Diagnostic criteria were enrolled in this study. PCOS patients were diagnosed when they fulfilled two of the following three criteria: (1) Clinical hyperandrogenism (Ferriman-Gallwey Score > 8) or Biochemical hyperandrogenism (elevated total/free testosterone); (2) Oligomenorrhea (less than 6-9 menses per year) or oligo-ovulation; (3) Polycystic ovaries on ultrasound ( $>=12$ Antral Follicles in one ovary or ovarian volume $>=10 \mathrm{~cm}^{3}$ ). Another 43 age-matched healthy women were recruited as control. In these participants, we have excluded the patients who have received special medication. Informed consent was signed and collected from all participants prior to the study. For the collection of PCOS ovary tissues, the ovarian drilling was performed with general anesthesia for laparoscopy and under general anesthesia. Tissues were snap-frozen and kept at $-80^{\circ} \mathrm{C}$ until use.

\subsection{Assessment of clinical parameters}

Body mass index (BMI) was calculated as weight (kilograms) divided by the square of the height (square meters). Blood samples were collected in the morning between 8:30 AM and 10:30 AM after overnight 
fasting. Systolic BP $(\mathrm{mmHg})$, diastolic BP $(\mathrm{mmHg})$, pulse (times/min), fasting glucose $(\mathrm{mmol} / \mathrm{L})$, urea $(\mathrm{mmol} / \mathrm{L})$, creatinine $(\mu \mathrm{mol} / \mathrm{L})$, alkaline phosphatase (ALP, $\mathrm{U} / \mathrm{L})$, alanine aminotransferase $(A L T, U / L)$, cholesterol ( $\mathrm{mmol} / \mathrm{L})$, high-density lipoprotein $(\mathrm{HDL})$-cholesterol $(\mathrm{mmol} / \mathrm{L})$, triglycerides $(\mathrm{mmol} / \mathrm{L})$, lowdensity lipoprotein $(\mathrm{LDL})$-cholesterol $(\mathrm{mmol} / \mathrm{L})$ were measured as routine test.

\subsection{Detection of mtDNA copy number in peripheral blood and ovarian tissues by the real-time polymerase chain reaction}

Peripheral blood and ovarian tissues mtDNA was extracted by using Trizol. The relative mtDNA copy number was measured by a real-time polymerase chain reaction (QPCR). The measurement was conducted by following Sang-Hee Lee's methods [19]. Primers used in the study were as follows:

B-globin Forward: 5'- GAAGAGCCAAGGACAGGTAC-3'

Reverse: 5'- CAACTTCATCCACGTTCACC-3'

MT-ND1 Forward: 5'- AACATACCCATGGCCAACCT-3'

Reverse: 5'- AGCGAAGGGTTGTAGTAGCCC-3'

The Real-time polymerase chain reaction was performed under the following conditions: initial denaturation at $95^{\circ} \mathrm{C}$ for 300 seconds followed by 40 cycles of 0.1 second at $95^{\circ} \mathrm{C}, 6$ seconds at $58^{\circ} \mathrm{C}$, and 18 seconds at $72^{\circ} \mathrm{C}$, and the real-time polymerase chain reaction was performed with 8 seconds extension time when identifying the mitochondrial gene products. The $\beta$-globin gene was used as a housekeeping gene. To reduce variations in measurements, all parameters throughout the study were measured by the same person.

\subsection{Statistical analysis}

All data were analyzed using the SPSS 25 statistical program and $\mathrm{P}<0.05$ was considered statistically significant. Data are presented as mean \pm standard deviation. Measurements with a skewed distribution were normalized by logarithmic transformation. The T-test was used to analyze the differences between PCOS patients and control participants. Pearson correlation coefficients were calculated, and logistic regression analyses were performed to evaluate the relationship between log-transformed mtDNA copy number and clinical characteristics in both the PCOS and control groups.

\section{Abbreviations}




\begin{tabular}{ll} 
PCOS & Polycystic ovary syndrome \\
\hline ROS & Reactive oxygen species \\
\hline mtDNA & mitochondrial DNA
\end{tabular}

\section{Declarations}

\section{Availability of data and material}

The datasets generated during the current study are available from the corresponding author on reasonable request.

\section{Acknowledgements}

Not applicable.

\section{Funding}

This work was supported by Health and Medical Research Fund which is funded by the Hong Kong government (HMRF) (04152646). Guangzhou Science and Technology Program Project (202002030173), Panyu District Key Medical and Health Discipline Project (2019-Z04-09).

\section{Author Contributions}

Conceptualization, T.T. and Y.S.; methodology, Y.S., Y.D. and T.T.; formal analysis, Y.S. and H.L.; writingoriginal draft preparation, Y.S and Y.D.; writing-review and editing, T.Y.,T.T., T.C.L. and C.C.W.; supervision, P.W.C. ; funding acquisition, T.T. All authors have read and agreed to the published version of the manuscript.

\section{Ethics approval and consent to participate}

This study was approved by the Institutional Review Board at the Chinese University of Hong Kong (CREC2016.663). All the consent forms were signed and collected prior to sample collection.

\section{Consent for publication}

Patients agree to participate in this work.

\section{Competing interests}

No competing interests declared.

\section{References}


1. Asunción, M.; Calvo, R.M.; San Millán, J.L.; Sancho, J.; Avila, S.; Escobar-Morreale, H.F. A prospective study of the prevalence of the polycystic ovary syndrome in unselected Caucasian women from Spain. The Journal of clinical endocrinology and metabolism 2000, 85, 2434-2438, doi:10.1210/jcem.85.7.6682.

2. Azziz, R.; Woods, K.S.; Reyna, R.; Key, T.J.; Knochenhauer, E.S.; Yildiz, B.O. The prevalence and features of the polycystic ovary syndrome in an unselected population. The Journal of clinical endocrinology and metabolism 2004, 89, 2745-2749, doi:10.1210/jc.2003-032046.

3. March, W.A.; Moore, V.M.; Willson, K.J.; Phillips, D.I.; Norman, R.J.; Davies, M.J. The prevalence of polycystic ovary syndrome in a community sample assessed under contrasting diagnostic criteria. Human reproduction (Oxford, England) 2010, 25, 544-551, doi:10.1093/humrep/dep399.

4. Chung, P.W.; Chan, S.S.; Yiu, K.W.; Lao, T.T.; Chung, T.K. Menstrual disorders in a Paediatric and Adolescent Gynaecology Clinic: patient presentations and longitudinal outcomes. Hong Kong medical journal = Xianggang yi xue za zhi 2011, 17, 391-397.

5. Ovesen, P.G.; Møller, N.; Greisen, S.; Ingerslev, H.J. [Polycystic ovary syndrome II. Endocrinology and metabolism]. Ugeskrift for laeger 1998, 160, 265-269.

6. Homburg, R. Polycystic ovary syndrome - from gynaecological curiosity to multisystem endocrinopathy. Human reproduction (Oxford, England) 1996, 11, 29-39, doi:10.1093/oxfordjournals.humrep.a019031.

7. Revised 2003 consensus on diagnostic criteria and long-term health risks related to polycystic ovary syndrome. Fertility and sterility 2004, 81, 19-25, doi:10.1016/j.fertnstert.2003.10.004.

8. Azziz, R.; Carmina, E.; Dewailly, D.; Diamanti-Kandarakis, E.; Escobar-Morreale, H.F.; Futterweit, W.; Janssen, O.E.; Legro, R.S.; Norman, R.J.; Taylor, A.E., et al. The Androgen Excess and PCOS Society criteria for the polycystic ovary syndrome: the complete task force report. Fertility and sterility 2009, 91, 456-488, doi:10.1016/j.fertnstert.2008.06.035.

9. Wild, R.A.; Carmina, E.; Diamanti-Kandarakis, E.; Dokras, A.; Escobar-Morreale, H.F.; Futterweit, W.; Lobo, R.; Norman, R.J.; Talbott, E.; Dumesic, D.A. Assessment of cardiovascular risk and prevention of cardiovascular disease in women with the polycystic ovary syndrome: a consensus statement by the Androgen Excess and Polycystic Ovary Syndrome (AE-PCOS) Society. The Journal of clinical endocrinology and metabolism 2010, 95, 2038-2049, doi:10.1210/jc.2009-2724.

10. Norman, R.J.; Dewailly, D.; Legro, R.S.; Hickey, T.E. Polycystic ovary syndrome. Lancet (London, England) 2007, 370, 685-697, doi:10.1016/s0140-6736(07)61345-2.

11. Hart, R.; Doherty, D.A. The potential implications of a PCOS diagnosis on a woman's long-term health using data linkage. The Journal of clinical endocrinology and metabolism 2015, 100, 911-919, doi:10.1210/jc.2014-3886.

12. Mather, K.J.; Kwan, F.; Corenblum, B. Hyperinsulinemia in polycystic ovary syndrome correlates with increased cardiovascular risk independent of obesity. Fertility and sterility 2000, 73, 150-156, doi:10.1016/s0015-0282(99)00468-9. 
13. Cascella, T.; Palomba, S.; De Sio, I.; Manguso, F.; Giallauria, F.; De Simone, B.; Tafuri, D.; Lombardi, G.; Colao, A.; Orio, F. Visceral fat is associated with cardiovascular risk in women with polycystic ovary syndrome. Human reproduction (Oxford, England) 2008, 23, 153-159, doi:10.1093/humrep/dem356.

14. Dan Dunn, J.; Alvarez, L.A.; Zhang, X.; Soldati, T. Reactive oxygen species and mitochondria: A nexus of cellular homeostasis. Redox biology 2015, 6, 472-485, doi:10.1016/j.redox.2015.09.005.

15. Annesley, S.J.; Fisher, P.R. Mitochondria in Health and Disease. Cells 2019, 8, doi:10.3390/cells8070680.

16. Harman, D. The biologic clock: the mitochondria? Journal of the American Geriatrics Society 1972, 20, 145-147, doi:10.1111/j.1532-5415.1972.tb00787.x.

17. Skov, V.; Glintborg, D.; Knudsen, S.; Jensen, T.; Kruse, T.A.; Tan, Q.; Brusgaard, K.; Beck-Nielsen, H.; $\mathrm{H} \varnothing \mathrm{jlund}, \mathrm{K}$. Reduced expression of nuclear-encoded genes involved in mitochondrial oxidative metabolism in skeletal muscle of insulin-resistant women with polycystic ovary syndrome. Diabetes 2007, 56, 2349-2355, doi:10.2337/db07-0275.

18. Mecocci, P.; MacGarvey, U.; Kaufman, A.E.; Koontz, D.; Shoffner, J.M.; Wallace, D.C.; Beal, M.F. Oxidative damage to mitochondrial DNA shows marked age-dependent increases in human brain. Annals of neurology 1993, 34, 609-616, doi:10.1002/ana.410340416.

19. Lee, S.H.; Chung, D.J.; Lee, H.S.; Kim, T.J.; Kim, M.H.; Jeong, H.J.; Im, J.A.; Lee, D.C.; Lee, J.W. Mitochondrial DNA copy number in peripheral blood in polycystic ovary syndrome. Metabolism: clinical and experimental 2011, 60, 1677-1682, doi:10.1016/j.metabol.2011.04.010.

20. Yang, P.K.; Chou, C.H.; Chang, C.H.; Chen, S.U.; Ho, H.N.; Chen, M.J. Changes in peripheral mitochondrial DNA copy number in metformin-treated women with polycystic ovary syndrome: a longitudinal study. Reproductive biology and endocrinology : RB\&E 2020, 18, 69, doi:10.1186/s12958-020-00629-5.

21. Ajmal, N.; Khan, S.Z.; Shaikh, R. Polycystic ovary syndrome (PCOS) and genetic predisposition: A review article. European journal of obstetrics \& gynecology and reproductive biology: X 2019, 3, 100060, doi:10.1016/j.eurox.2019.100060.

22. Ilie, I.R. Advances in PCOS Pathogenesis and Progression-Mitochondrial Mutations and Dysfunction. Advances in clinical chemistry 2018, 86, 127-155, doi:10.1016/bs.acc.2018.05.003.

23. Zhang, J.; Bao, Y.; Zhou, X.; Zheng, L. Polycystic ovary syndrome and mitochondrial dysfunction. Reproductive biology and endocrinology : RB\&E 2019, 17, 67, doi:10.1186/s12958-019-0509-4.

24. Saeed, N.; Hamzah, I.H.; Al-Gharrawi, S.A.R. Polycystic ovary syndrome dependency on mtDNA mutation; copy Number and its association with insulin resistance. BMC research notes 2019, 12, 455, doi:10.1186/s13104-019-4453-3.

25. Shukla, P.; Mukherjee, S.; Patil, A. Identification of Variants in Mitochondrial D-Loop and OriL Region and Analysis of Mitochondrial DNA Copy Number in Women with Polycystic Ovary Syndrome. DNA and cell biology 2020, 39, 1458-1466, doi:10.1089/dna.2019.5323.

26. Ding, Y.; Zhuo, G.; Zhang, C. The Mitochondrial tRNALeu(UUR) A3302G Mutation may be Associated With Insulin Resistance in Woman With Polycystic Ovary Syndrome. Reproductive sciences 
(Thousand Oaks, Calif.) 2016, 23, 228-233, doi:10.1177/1933719115602777.

27. Papadakis, G.; Kandaraki, E.; Papalou, O.; Vryonidou, A.; Diamanti-Kandarakis, E. Is cardiovascular risk in women with PCOS a real risk? Current insights. Minerva endocrinologica 2017, 42, 340-355, doi:10.23736/s0391-1977.17.02609-8.

28. Boulman, N.; Levy, Y.; Leiba, R.; Shachar, S.; Linn, R.; Zinder, O.; Blumenfeld, Z. Increased C-reactive protein levels in the polycystic ovary syndrome: a marker of cardiovascular disease. The Journal of clinical endocrinology and metabolism 2004, 89, 2160-2165, doi:10.1210/jc.2003-031096.

29. Mohammadi, M. Oxidative Stress and Polycystic Ovary Syndrome: A Brief Review. International journal of preventive medicine 2019, 10,86, doi:10.4103/ijpvm.IJPVM_576_17.

30. Mobeen, H.; Afzal, N.; Kashif, M. Polycystic Ovary Syndrome May Be an Autoimmune Disorder. Scientifica 2016, 2016, 4071735, doi:10.1155/2016/4071735.

31. Cree, L. M., Patel, S. K., Pyle, A., Lynn, S., Turnbull, D. M., Chinnery, P. F., \& Walker, M. Age-related decline in mitochondrial DNA copy number in isolated human pancreatic islets. Diabetologia 2008, 51(8), 1440-1443.

32. LI, Zhihua, et al. Genetic variants in nuclear DNA along with environmental factors modify mitochondrial DNA copy number: a population-based exome-wide association study. BMC genomics, 2018, 19.1: 1-9.

\section{Figures}

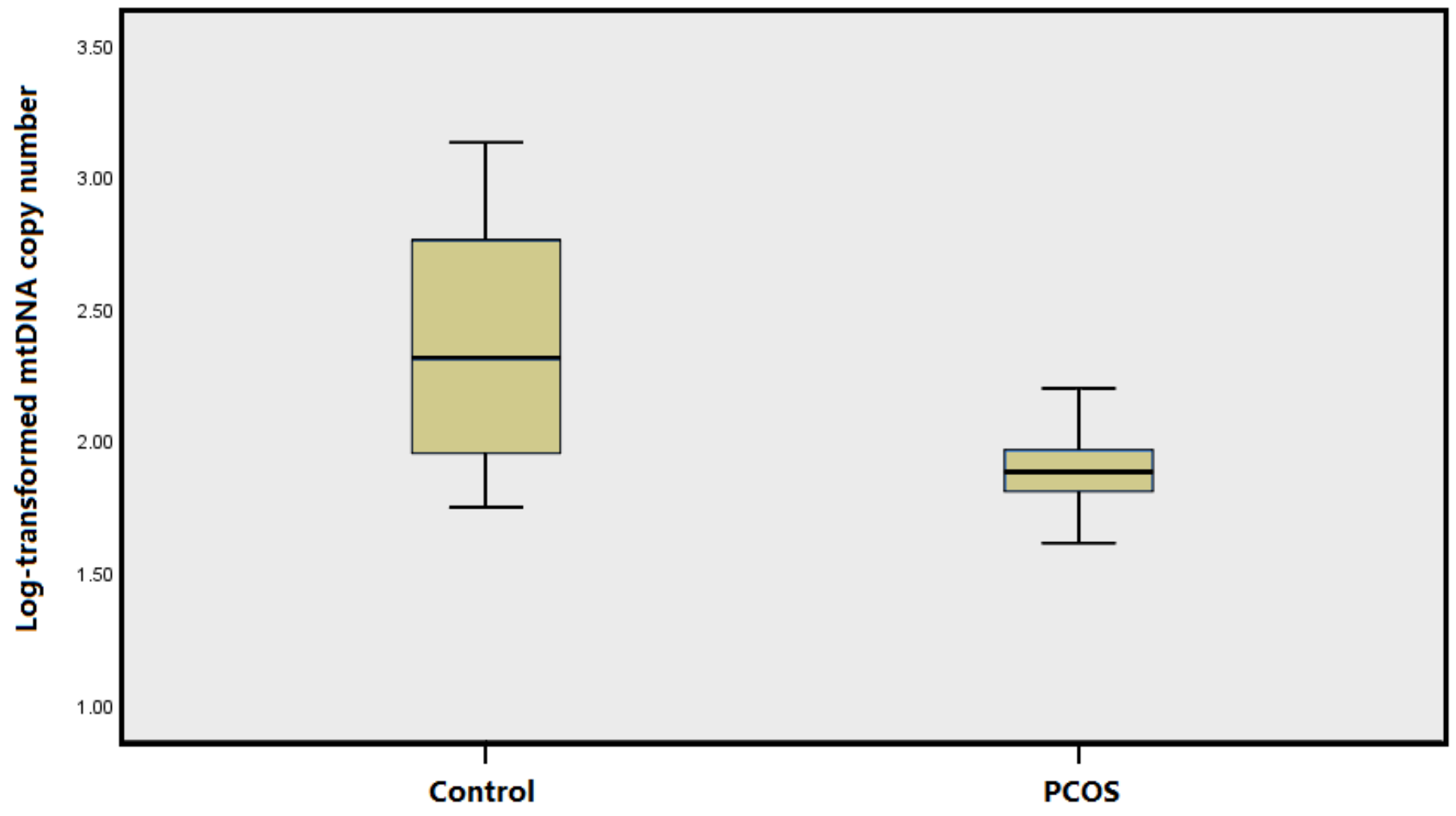


Figure 1

Log-transformed mtDNA copy number in peripheral blood from PCOS patients and control group

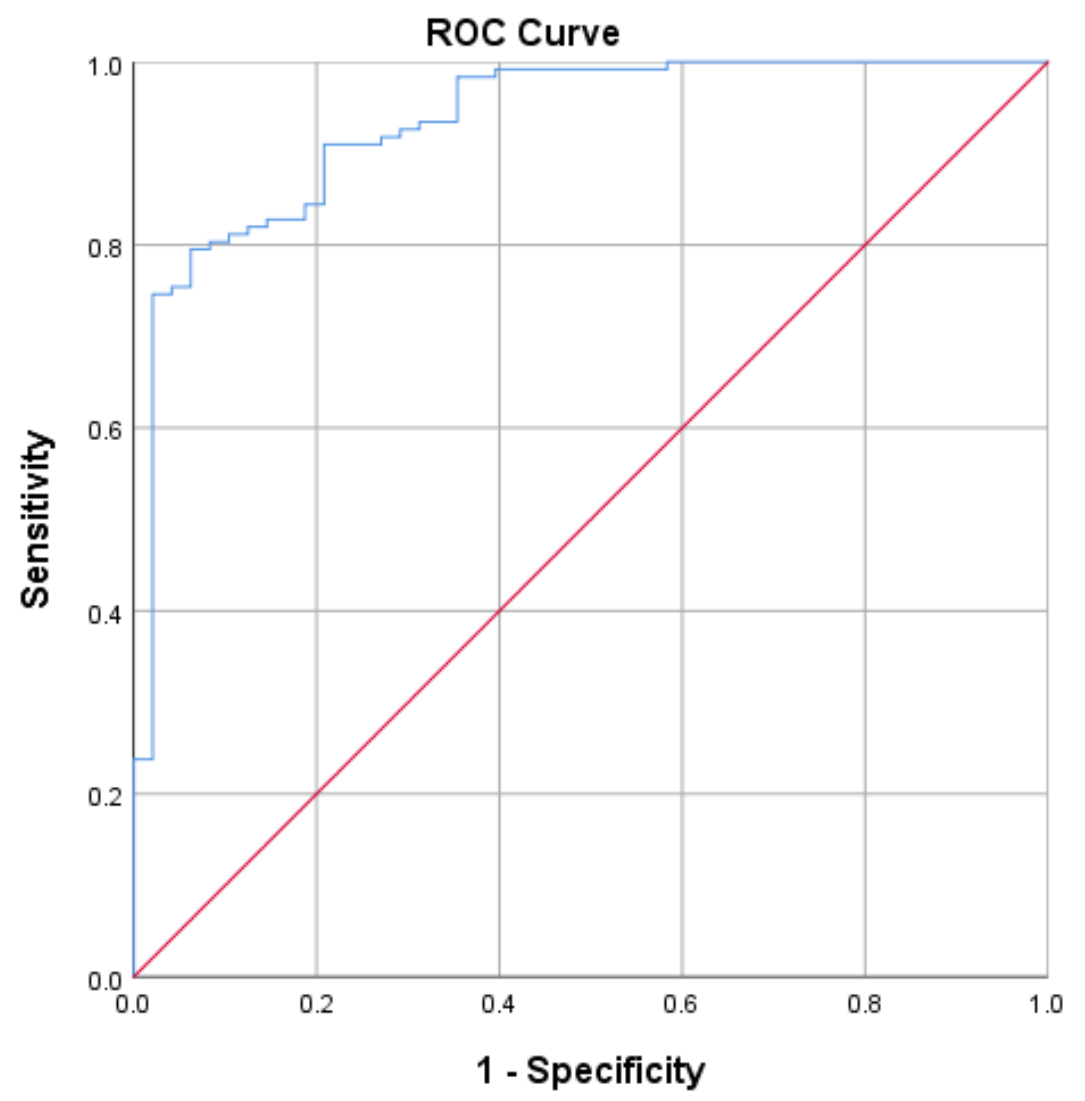

Figure 2

ROC curve for PCOS diagnosis (including BMI, pulse and mtDNA copy number) 